\title{
Large-Time Behavior of Solutions of Initial and Initial-Boundary Value Problems of a General System of Hyperbolic Conservation Laws`
}

\section{Tai-Ping Liu ${ }^{\star}$}

Courant Institute of Mathematical Sciences, New York University, New York, NY 10012, USA

\begin{abstract}
We study the asymptotic behavior of the solution of the initial and initial-boundary value problem of hyperbolic conservation laws when the initial and boundary data have bounded total variation. It is shown that the solution converges to the linear superposition of traveling waves, shock waves and rarefaction waves. The strength and speed of these waves depend only on the values of the data at infinity.
\end{abstract}

\section{§1. Introduction}

We consider a system of conservation laws

$$
U_{t}+F(U)_{x}=0 \text {, }
$$

where $F(U)$ and $U$ are $n$-vectors, $F=\left(F_{1}, \ldots, F_{n}\right), U=\left(U_{1}, \ldots, U_{n}\right), x \in R$ and $t \geqq 0$. We assume that the system is strictly hyperbolic and each characteristic field is either genuinely-non-linear or linearly degenerate in the sense of Lax [10]. We study the Cauchy problem (1.1) with initial data

$$
U(x, 0)=U_{0}(x)
$$

which is assumed to have bounded total variation so that the limiting values of $U_{0}$ at $x= \pm \infty$ exist:

$$
U_{l} \equiv U_{0}(-\infty), \quad U_{r} \equiv U_{0}(+\infty) .
$$

Our main purpose is to compare the solution $U(x, t)$ of $(1.1),(1.2)$ with the solution $U_{*}(x, t)$ of the corresponding Riemann problem (1.1) with

$$
U(x, 0)=\left\{\begin{array}{lll}
U_{l} & \text { for } & x<0 \\
U_{r} & \text { for } & x>0
\end{array}\right.
$$

* Results obtained at the Courant Institute of Mathematical Sciences, New York University while the author was a Visiting Member at the Institute; this work was supported by the National Science Foundation, Grant NSF-MCS 76-07039

$\star \star$ On leave from the University of Maryland, College Park, USA 
We show that $U(x, t)$ converges to $U_{*}(x, t)$ as $t$ tends to infinity in the following sense: In the primary $i$-th region, (5.1), all $j$-th waves, $i \neq j$, decay, (Theorem 5.2). If the $i$-th wave in $U_{*}(x, t)$ is a shock wave, then an $i$-th shock wave will appear in $U(x, t)$ as $t$ becomes large, and will approach the corresponding $i$-th shock in $U_{*}(x, t)$ and dominates $U(x, t)$ in the primary $i$-th region, [Theorem 5.7,(iii)]. If the $i$-th wave in $U_{*}(x, t)$ is a rarefraction wave, then all $i$-th shocks in $U(x, t)$ decay to zero and $U(x, t)$ approaches $U_{*}(x, t)$ in the primary $i$-th region, [Theorem 5.7, (ii)]. When the $i$-th wave in $U_{*}(x, t)$ is a contact discontinuity, i.e. when the $i$-th characteristic field is linearly degenerate, then all $i$-th waves have speed approaching that of the contact discontinuity of $U_{*}(x, t)$, in other words, the $i$-th waves become increasingly linear.

In particular if $U_{l}=U_{r}$, then all $i$-th waves decay except those associated with linear degenerate characteristic fields and thus if $\eta$ is a Riemann invariant for all linear degenerate fields, then $\eta$ tends to a constant. If, moreover, the system is genuinely nonlinear in all characteristic fields, then the solution decays to the constant $U_{l}=U_{r}$.

Our main assumption is that the total amount of interactions is finite (cf. Section 3). We carry out our analysis with Glimm's difference scheme, [5]. The scheme has a stochastic feature. By a compactness argument based on Helly's theorem [5], it is shown that the approximate solution $U_{h}$ converge to an exact solution $U$ if

$$
\text { total } \operatorname{var}_{x} U_{h}(x, t) \leqq \text { const total } \operatorname{var}_{x} U_{h}(x, 0)
$$

for some constant independent of $t$. When the initial data have small total variation, the estimate (1.4) was established in Glimm [5] by introducing a nonlinear functional defined on the approximate solutions. The functional consists of a linear and a quadratic term. The quadratic term measures the potential amount of interactions. It follows from the boundedness of the functional that the total amount of interactions is bounded. Thus our results apply for the Glimm solutions. Estimate (1.4) has also been established for certain systems where initial data need not be of small total variation, $[1,3,14,15,17,18,19]$. In particular, Nishida [17] solves the Cauchy problem for the model equations of gas dynamics

$$
\begin{aligned}
u_{t}-p(v)_{x} & =0, \\
v_{t}-u_{x} & =0, \quad p(v)=\mathrm{const} v^{-1}
\end{aligned}
$$

when the initial data have arbitrary finite total variation. For isentropic equations of a polytropic gas, $p(v)=$ const $v^{-\gamma}, \gamma>1$, Nishida and Smoller [18] obtain the uniform bound (1.4) under the assumption that $(\gamma-1)$ total $\operatorname{var}_{x} U(x, 0)$ is less than a constant independent of $\gamma$. Solutions for general equations of a polytropic gas have been constructed by Liu [14] under similar hypothesis. The functionals used in $[14,18]$ contain quadratic terms. Other aforementioned works do not use functionals containing quadratic terms, nevertheless we will show that for those solutions the total amount of interactions are finite and our results apply.

Our methods also apply to solutions of initial-boundary value problems in the quadrant $x \geqq 0, t \geqq 0$. It is shown that the asymptotic behavior of the solution is determined by the initial data at $x=+\infty$ and the boundary data at $t=+\infty$. We will illustrate this for general gas equations, Theorem 6.1 . The initial-boundary value 
problems for general gas equations with pressures or velocity given at $x=0$ have been studied by Liu [15].

The theory of decay for genuinely nonlinear systems of conservation laws has been developed by Glimm and Lax [6]. The Glimm-Lax theory is developed for systems of two conservation laws when initial data have small oscillation. In the case when the initial data are constant outside a finite interval, the solution decay to zero at the rate $t^{-1 / 2}$. With periodic initial data, the total variation of the solution per period decays uniformly at the rate $t^{-1}$. When the initial data equal $U_{l}$ for $x<-N$ and $U_{r}$ for $x>N$ for some $N>0$, and the solution contains only weak shock waves, it was shown by Liu [16] that the solution of (1.1), (1.2) converges to the solution of (1.1), (1.3) at algebraic rates.

Our results on the asymptotic behavior of solutions of general systems of $n$ conservation laws, $n \geqq 2$, reduces to results on the decay of solutions when the initial data are constant outside a finite interval. Such decay results have been obtained by DiPerna [4] under an additional assumption that system (1.1) possesses a convex extension in the sense of Lax [11]. Since we do not assume the initial data to be constant for $|x|$ large, we do not expect the solution to converge at algebraic rates. When $U_{0}(x)$ equals $U_{l}$ for $x<-N$, and $U_{r}$ for $x>N, N>0$, we believe that the solution converges to that of the corresponding Riemann problem at algebraic rates. However, new techniques are required for the proof; we leave this for the future. For the asymptotic behavior of special solutions see $[2,7]$ and $[8]$.

The primary reasons for the simple large-time behavior of the solution are the spreading of rarefaction waves which forces the cancellation of shock and rarefaction waves of the same genuinely nonlinear characteristic field. Waves of the same linearly degenerate family do not cancel and behave like linear waves. For general systems, the interaction of waves may change the speeds and magnitudes of waves and may produce new waves. Since we assume the total amount of interaction to be finite, the amount of interaction after large time is small. It follows that the solution is almost uncoupled, (Lemma 5.1). We then use the asymptotic results for scalar equations, Liu [16], to show that the solution approaches that of the corresponding Riemann problem. Our main tool is the theory of generalized characteristics developed by Glimm and Lax [6].

It is essential for our methods that the Riemann problem has a unique solution. For general systems of conservation laws, Lax [11] uses the implicit function theorem to show that the Riemann problem has a unique self-similar solution in a small neighborhood of a constant. For a wide class of two-conservation laws, Smoller $[21,22]$ solved the Riemann problem when the initial states may not be close. The Riemann problem for general gas equations has been solved by Liu [13] and Smith [20] for arbitrary initial states. In proving that the system is increasingly uncoupled, we assume that the characteristic speeds are strictly separated for any approximate solutions under consideration, [(5.1)]. This assumption is satisfied for nearconstant solutions of general systems and also solutions of general gas equations in the Lagrangian coordinates which are bounded away from the vacuum. This assumption can be relaxed, however.

The space of functions of bounded variation is a natural space for the solution operator of a system of conservation laws. Even if the initial data are analytic, in general the solution is not smooth due to the nonlinearity of the system. On the 
other hand, results on decay and asymptotic behavior of solutions show that the nonlinearity of the system has certain smoothing effects, and these results may be viewed as results on the regularity of the solutions.

In the next section we will describe briefly the Riemann problem, the Glimm difference scheme, Glimm and Lax's notions of approximate conservation laws. In Section 3 we investigate the assumption on the boundedness of total amount of interactions for existing existence theorems. Section 4 studies the spreading of rarefaction waves. The main results on the asymptotic behavior of solutions of initial value problems are proved in Section 5. The initial-boundary value problems for gas equations are studied in Section 6.

\section{§2. Preliminary}

We assume that system (1.1) is strictly hyperbolic, i.e. $\partial F(U) / \partial U$ has real and distinct eigenvalues $\lambda_{1}(U)<\lambda_{2}(U)<\ldots<\lambda_{n}(U)$. Assume that each characteristic field is either genuinely nonlinear or linearly degenerate, i.e. for any $U$ under consideration,

$$
\begin{array}{ll}
r_{\alpha_{i}}(U) \cdot \nabla_{U} \lambda_{\alpha_{i}}(U) \neq 0, & i=1,2, \ldots, p, \\
r_{\beta_{j}}(U) \cdot \nabla_{U} \lambda_{\beta_{j}}(U) \equiv 0, & j=1,2, \ldots, n-p,
\end{array}
$$

where $r_{i}(U), i=1,2, \ldots, n$, is an $i$-th right eigenvector of $\partial F(U) / \partial U$ and $\left\{\alpha_{1}, \alpha_{2}, \ldots, \alpha_{p}\right.$, $\left.\beta_{1}, \beta_{2}, \ldots, \beta_{n-p}\right\}=\{1,2, \ldots, n\}$. The rarefaction curve $R_{i}\left(U_{0}\right), i=1,2, \ldots, n$ is the integral curve of $r_{i}$ through the point $U_{0}$; and the shock curve $S_{i}\left(U_{0}\right), i=1,2, \ldots, n$, is a curve tangent to $R_{i}\left(U_{0}\right)$ at $U_{0}$ and for all $U \in U_{0},\left(U_{0}, U\right)$ satisfies the following Rankine-Hugoniot condition

$$
\sigma\left(U, U_{0}\right)\left(U-U_{0}\right)=F(U)-F\left(U_{0}\right)
$$

for some scalar $\sigma=\sigma\left(U_{0}, U\right)$, the shock speed for $\left(U_{0}, U\right)$. When $i \in\left\{\beta_{1}, \ldots, \beta_{n-p}\right\}$, $R_{i}\left(U_{0}\right)=S_{i}\left(U_{0}\right),(\operatorname{Lax}[11])$, and for any $U \in R_{i}\left(U_{0}\right), \sigma\left(U_{0}, U\right)=\lambda_{i}\left(U_{0}\right)=\lambda_{i}(U), U_{0}$ is connected to $U$ by an $i$-th contact discontinuity. For $i \in\left\{\alpha_{1}, \ldots, \alpha_{p}\right\}$ and $U \in R_{i}^{+}\left(U_{0}\right) \equiv\left\{U \in R_{i}\left(U_{0}\right) \mid \lambda_{i}(U)>\lambda_{i}\left(U_{0}\right)\right\}$, then $U_{0}$ can be connected to $U$ on the right by an $i$-th rarefaction wave; if $U \in S_{i}^{-}\left(U_{0}\right) \equiv\left\{U \in S_{i}\left(U_{0}\right) \mid \lambda_{i}(U)<\lambda_{i}\left(U_{0}\right)\right\}$, then $U_{0}$ can be connected to $U$ on the right by an $i$-th shock wave satisfying the shock inequality of Lax [11]:

$$
\lambda_{i}(U)<\sigma\left(U_{0}, U\right)<\lambda_{i}\left(U_{0}\right) .
$$

We set

$$
T_{i}\left(U_{0}\right)= \begin{cases}R_{i}\left(U_{0}\right)=S_{i}\left(U_{0}\right), & \text { for } i \in\left\{\beta_{1}, \ldots, \beta_{n-p}\right\}, \\ S_{i}^{-}\left(U_{0}\right) U R_{i}^{+}\left(U_{0}\right), & \text { for } i \in\left\{\alpha_{1}, \ldots, \alpha_{p}\right\},\end{cases}
$$

so that $U_{0}$ can be connected to any $U$ on $T_{i}\left(U_{0}\right)$ on the right by an $i$-th wave.

The Riemann problem (1.1), (1.3) is solved by finding $U_{i}, i=0,1,2, \ldots, n, U_{0}=U_{l}$, $U_{n}=U_{r}, U_{i} \in T_{i}\left(U_{i-1}\right)$ so that $U_{i-1}$ is connected to $U_{i}$ on the right by a centered $i$-th wave, denoted as $\left(U_{i-1}, U_{i}\right)$. Solutions of Riemann problems are the building blocks for the Glimm's difference scheme, [5]. Let $r, s$ be mesh lengths so chosen that $r / s \geqq \max \left\{\lambda_{i}\right\}$. The Glimm's approximate solution $U_{s}(x, t)$ is exact in the strip $n s \leqq t$ $\leqq(n+1) s$ and consists of elementary waves generated at $t=n s, x=m r, m+n=$ even. 
At time $(n+1) s$, the value of $U_{s}(x, t)$ in the interval $(m-1) r<x<(m+1) r$ is set to be the value of the exact solution constructed in the strip $t=(n+1) s$ and $x=\left(m+\alpha_{n+1}\right) r$. Here $\left\{\alpha_{n}\right\}$ is a randomly chosen sequence, equidistributed in $(-1,1)$. An $I$-curve is a space-like curve consisting of a segment joining neighboring mesh points $\left(\left(m+\alpha_{n}\right) r, n s\right), m+n=$ even. The upper half plane $t \geqq 0$ is covered by diamonds $\Delta_{n, m}$ with vertices $\left(\left(m+\alpha_{n-1}\right) r,(n-1) s\right),\left(\left(m+\alpha_{n+1}\right) r,(n+1) s\right),\left(\left(m-1-\alpha_{n}\right) r, n s\right)$, $\left(\left(m+1+\alpha_{n}\right) r, n s\right)$.

The strength of the $i$-th wave $\left(U_{i-1}, U_{i}\right)$ in the solution of the Riemann problem $\left(U_{l}, U_{r}\right)$ is defined as

$$
\left(U_{l}, U_{r}\right)_{i} \equiv w_{i}\left(U_{i-1}\right)-w_{i}\left(U_{i}\right), \quad i=1,2, \ldots, n,
$$

where $w_{i}=\lambda_{i}$ if $i=\alpha_{1}, \ldots, \alpha_{p}$ and $w_{i}$ is any increasing function along $T_{i}$ for $i=\beta_{1}, \ldots, \beta_{n-p}$. The first step in establishing estimates such as (1.4) is to investigate the interaction of waves of solutions of two Riemann problems. Suppose that the Riemann problems $\left(U_{l}, U_{m}\right),\left(U_{m}, U_{r}\right)$ and $\left(U_{l}, U_{r}\right)$ can be solved. Then in rather general circumstances, there exists a quantity $Q\left(U_{l}, U_{m}, U_{r}\right)$, the potential amount of interactions, so that for some constant $0(1)$ depending only on the system (1.1),

$$
\left(U_{l}, U_{r}\right)_{i}=\left(U_{l}, U_{m}\right)_{i}+\left(U_{m}, U_{r}\right)_{i}+0(1) Q\left(U_{l}, U_{m}, U_{r}\right) .
$$

Given any diamond $\Delta$ in the Glimm scheme, if the waves entering $\Delta$ from the right and left as solutions of the Riemann problems $\left(U_{m}, U_{r}\right)$ and $\left(U_{l}, U_{m}\right)$ respectively, then we set $Q(\Delta)=Q\left(U_{l}, U_{m}, U_{r}\right)$. We also set the amount of cancellation in $\Delta$ as

$$
C_{i}(\Delta)=\frac{1}{2}\left[\left|\left(U_{l}, U_{m}\right)\right|_{i}+\left|\left(U_{m}, U_{r}\right)_{i}\right|-\left|\left(U_{l}, U_{m}\right)_{i}+\left(U_{m}, U_{r}\right)_{i}\right|\right] \text {. }
$$

Let $\Lambda$ be a collection of diamonds. We denote by $E_{i}^{+}(\Lambda)$ and $E_{i}^{-}(\Lambda)$ the total amount of $i$-th rarefaction and shock waves, respectively, entering $\Lambda$. The amount of waves leaving $\Lambda$ is denoted by $L_{i}^{ \pm}(\Lambda)$. Summing up (2.5) for all diamonds in $\Lambda$ we obtain the following approximate conservation laws

$$
L_{i}^{ \pm}(\Lambda)=E_{i}^{ \pm}(\Lambda) \mp C_{i}(\Lambda)+0(1) Q(\Lambda),
$$

where $C_{i}(\Lambda)=\sum_{\Delta \in \Lambda} C_{i}(\Delta), Q(\Lambda)=\sum_{\Delta \in \Lambda} Q(\Delta)$. In the next section we will investigate the amount of interactions $Q$.

\section{§3. The Amount of Interactions}

For a general system of $n$-conservation laws, Glimm [5] obtains the following estimate for any nearby states $U_{l}, U_{m}, U_{r}$ :

$$
\left(U_{l}, U_{r}\right)_{i}=\left(U_{l}, U_{m}\right)_{i}+\left(U_{m}, U_{r}\right)_{i}+O(1) D\left(U_{l}, U_{m}, U_{r}\right)
$$

where $D\left(U_{l}, U_{m}, U_{r}\right)$ is the sum of products of the strength of approaching waves. An $i$-th wave $\alpha$ approaches a $j$-th wave $\beta$ if either $i>j$ and $\alpha$ lies toward the left of $\beta$, or $i=j$ and at least one of $\alpha$ and $\beta$ is a shock wave. Given any $I$-curve $J$, we define $D(J)$ as the sum of products of strength of approaching waves which cross $J$. If $J_{2}$ is an immediate successor of $J_{1}$, i.e. $J_{1}$ and $J_{2}$ sandwich a diamond $\Delta$ and $J_{2}$ lies toward larger time than $J_{1}$, then it follows directly that

$$
D\left(J_{2}\right)-D\left(J_{1}\right) \leqq-D(\Delta)+O(1) L\left(J_{1}\right) D(\Delta)
$$


where $L\left(J_{1}\right)$ is the total amount of waves crossing $J_{1}$. Thus for $L\left(J_{1}\right)$ small enough $D\left(J_{2}\right)-D\left(J_{1}\right) \leqq-\frac{1}{2} D(\Delta)$. If we sum up this inequality over all diamonds in a region $\Lambda$, one gets

$$
D(\Lambda) \leqq 2 D(J)
$$

for any $I$-curve $J$ containing the domain of dependence of $\Lambda$. This shows in particular if $L(J)$ is small, then $D(\Lambda)$ is bounded for all $\Lambda$. Thus the total mount of interaction $D(\Lambda)$ is finite if the initial data have small total variation.

For isentropic gas equations (1.5) with $p(v)=$ const $v^{-\gamma}, \gamma>1$, Nishida and Smoller [8] show that global solution exists if $(\gamma-1)$ times the total variation of the initial data is sufficiently small. Under this assumption it is not hard to see from their estimates that

$$
Q(\Lambda) \leqq \text { const } F(0)(\gamma-1)^{-1},
$$

where $F(J)=L(J)+(\gamma-1) D(J)$ and $L(J)$ is the total mount of shock waves crossing $J$, $D(J)$ is a quadratic term measuring the potential amount of interaction. The inequality (3.3) is obtained from

$$
F\left(J_{2}\right)-F\left(J_{1}\right) \leqq-\frac{(\gamma-1)}{2} D(\Delta) .
$$

Estimate (3.3) is crude when $\gamma$ is close to 1 . Suppose that the strength of waves is measured by a linear combination of Riemann invariants (cf. Liu [15]) and we set

$$
\begin{aligned}
\tilde{F}(J)= & \tilde{L}(J)+\tilde{F}(J), \\
\tilde{L}(J)= & \text { total mount of waves crossing } J, \\
\tilde{D}(J)= & K \sum_{J}\{\mid \alpha \beta \| \alpha \text { and } \beta \text { are strengths of approaching shock waves }\} \\
& +H \sum_{J}\{|\alpha \beta| \alpha \text { and } \beta \text { are strengths of approaching waves and not both are } \\
& \text { shock waves }\},
\end{aligned}
$$

where $K$ and $H$ are constants independent of $\gamma$. Then if we choose $K$ and $H$ sufficiently small, $H$ small compared to $K$, it follows that

$$
\tilde{F}\left(J_{2}\right)-\tilde{F}\left(J_{1}\right) \leqq-\frac{1}{2} \tilde{D}(\Delta)
$$

whence we obtain an estimate stronger than (3.3):

$$
Q(\Lambda) \leqq \text { const } F(0) \text {. }
$$

The inequality (3.4) is proved by detailed analysis of wave interactions, [8]. Analogous estimate also holds for general gas equation, [15]. We omit the details.

The result of Nishida was generalized by Bakharov [1] where the existence theorem was proved under the assumption that shock strength does not increase after interaction. If one measures the strength of the wave not by Riemann invariants but instead by linear combination of Riemann invariants, [15], then after detailed analysis of wave interactions, one sees that

$$
F^{*}\left(J_{2}\right)-F^{*}\left(J_{1}\right) \leqq-D^{*}(\Delta) / L(0)
$$


where $D^{*}(\Delta)$ is given as in (3.1), $F^{*}=L^{*}+D^{*} / L(0)$ and $L^{*}(J)$ measures only the amount of shock wave crossing $J$. Thus one concludes by summing up (3.6) that

$$
Q(\Lambda) \leqq F(0) L(0) \leqq 2(L(0))^{2}
$$

where $L(0)$ is the total amount of shock waves for $I$-curve connecting points on $t=0$ and $t=s$ in Glimm's scheme. We thus obtain the estimate (3.7) which is as strong as (3.2) which holds only for solutions near the constant.

\section{§4. Expansion of Rarefaction Waves}

Generalized characteristics are Lipschitz continuous curves in the $x t$-space which propagate with either shock or characteristic speeds. Such curves can be constructed by the recipe of Glimm-Lax's [6]. The two-sided limits of the solution exist along a generalized characteristic except for a countable value of $t$. Given any $k$ characteristics $\chi_{k}^{1}$ and $\chi_{k}^{2}$ issued from time $t_{0}, k \in\{1,2, \ldots, n\}, \chi_{k}^{1}$ lies to the left of $\chi_{k}^{2}$, we set

$D_{k}(t)=$ distance between $\chi_{k}^{1}$ and $\chi_{k}^{2}$ at time $t, t \geqq t_{0}$,

$X_{k}^{ \pm}(t)=$ amount of $k$-rarefaction and $k$-shock waves, respectively, between (but not on) $\chi_{k}^{1}$ and $\chi_{k}^{2}$ at time $t$,

$\tilde{X}_{k}(t)=$ total amount of $j$-th waves, $j \neq k$, between $\chi_{k}^{1}$ and $\chi_{k}^{2}$ at time $t$,

$U_{k}^{ \pm i}(t)=$ the one-sided limit from right and left, respectively, of $U(x, t)$ at the point $(x, t)$ on $\chi_{k}^{i}, i=1,2$.

We now assume that the $k$-characteristic family is genuinely nonlinear so that we have

$$
\lambda_{k}^{+i}(t) \equiv \lambda_{k}\left(U_{k}^{+i}(t)\right) \leqq \sigma_{k}\left(U_{k}^{+i}(t), U_{k}^{-i}(t)\right) \leqq \lambda_{k}\left(U_{k}^{-i}(t)\right) \equiv \lambda_{k}^{-i}(t) .
$$

It follows easily from the Rankine-Hugoniot condition and the mean-value theorem that for some $\theta(t), 0<\theta(t)<1$,

$$
\begin{aligned}
\dot{D}_{k}(t) & =\sigma_{k}\left(U_{k}^{+2}(t), U_{k}^{-2}(t)\right)-\sigma_{k}\left(U_{k}^{+1}(t), U_{k}^{-2}(t)\right) \\
& =\theta(t)\left[\lambda_{k}^{-2}(t)-\lambda_{k}^{+1}(t)\right]+(1-\theta(t))\left[\lambda_{k}^{+2}(t)-\lambda_{k}^{-1}(t)\right] .
\end{aligned}
$$

We note that $\theta(t)=\theta\left(\lambda_{k}^{ \pm 1}(t), \lambda_{k}^{ \pm 2}(t)\right)$ and $\lambda_{k}^{ \pm 1}(t), \lambda_{k}^{ \pm 2}(t)$ range over a compact set in $U$ space. Thus there exists a constant $\theta, 0<\theta<1$, independent of $t$ such that

$$
\dot{D}_{k}(t) \leqq \theta\left[\lambda_{k}^{-2}(t)-\lambda_{k}^{+1}(t)\right]+(1-\theta)\left[\lambda_{k}^{+2}(t)-\lambda_{k}^{-1}(t)\right] .
$$

Since $\lambda_{k}^{-2}(t)-\lambda_{k}^{+1}(t)=X_{k}^{+}(t)+X_{k}^{-}(t)+0(1) \tilde{X}_{k}(t)$ as is easily seen, it follows from (4.2) that

$$
\begin{aligned}
\dot{D}_{k}(t)= & X_{k}^{+}(t)+X_{k}^{-}(t)+0(1) \tilde{X}_{k}(t) \\
& +(1-\theta(t))\left[\operatorname{str} \chi_{1}(t)+\operatorname{str} \chi_{2}(t)\right] .
\end{aligned}
$$

Since the characteristic speeds are assumed to be strictly separated, there exists a finite $t_{1}>t_{0}$ such that all $i$-th generalized characteristics $\chi_{i}^{1}$ and $\chi_{i}^{2}, i \neq k$, meet $\chi_{k}^{1}$ or $\chi_{k}^{2}$ before time $t_{1}$. Similarly, for any $t>t_{1}$, there exists $s<t$ such that the $(k-1)$-th 
$\left[(k+1)\right.$-th] generalized characteristics through a point on $\chi_{k}^{2}\left(\chi_{k}^{1}\right)$ at time $s$ meets $\chi_{k}^{1}\left(\chi_{k}^{2}\right)$ before time $t$, and for some 0(1) independent of $t$,

$$
t-s=0(1) D_{k}(t) \text {. }
$$

We denote by $h_{k}\left(t_{0}, t\right)$ the amount of $i$-th waves, $i \neq k$, crossing $X_{k}^{1}$ or $X_{k}^{2}$ between time $t_{0}$ and $t$. Let $Q\left(t_{0}, t\right)$ be the amount of interactions in the region between $\chi_{k}^{1}$ and $\chi_{k}^{2}$ between $t_{0}$ and $t$. We have from (2.7),

$$
\tilde{X}_{k}(t)=0(1) \int_{s}^{t} d\left(Q\left(t_{0}, \tau\right)+h_{k}\left(t_{0}, \tau\right)\right) .
$$

Integrating (4.4) from $t_{1}$ to $t, t>t_{1}$, and using (4.5), (4.6) we obtain

$$
\begin{aligned}
D_{k}(t) \leqq & D_{k}\left(t_{1}\right)+\int_{t_{1}}^{t}\left[X_{k}^{+}(\tau)+X_{k}^{-}(\tau)\right. \\
& \left.+(1-\theta(t))\left(\operatorname{str} \chi_{1}(\tau)+\operatorname{str} \chi_{2}(\tau)\right)\right] d \tau \\
& +0(1) \int_{t_{1}}^{t} D_{k}(\tau) d\left(Q\left(t_{0}, \tau\right)+h_{k}\left(t_{0}, \tau\right)\right) .
\end{aligned}
$$

Since $k$-th waves may cross $\chi_{k}^{1}$ or $\chi_{k}^{2}$ only due to interactions, we have from (2.7)

$$
\begin{aligned}
& X_{k}^{+}(s) \geqq X_{k}^{+}(t)-0(1) Q(s, t), \quad t_{0} \leqq s \leqq t, \\
& X_{k}^{-}(s) \geqq X_{k}^{-}\left(t_{0}\right)-0(1) Q\left(t_{0}, s\right),
\end{aligned}
$$

and thus we may solve the linear integral inequality (4.6) to obtain

$$
X_{k}^{+}(t) \leqq \frac{D_{k}(t)}{t-t_{1}}+0(1)\left[Q\left(t_{0}, t\right)+h_{k}\left(t_{0}, t\right)-X_{k}^{-}(t)-\max \operatorname{str}\right]
$$

where max str is the maximum strength of $\chi_{k}^{1}$ and $\chi_{k}^{2}$ between $t_{0}$ and $t$. We may apply this inequality to subregions which contain predominantly $k$-th rarefraction waves and the boundary of these subregions may be so chosen that it consists of $k$ characteristics with small strengths ([6], pp. 88-92). Thus the above inequality holds without the last two terms on the right. We list this as a theorem.

Theorem 4.1. Let $\chi_{k}^{1}$ and $\chi_{k}^{2}, k=1,2, \ldots, n$, be generalized $k$-characteristics issued from two points on $t=t_{0}, \chi_{k}^{1}$ lying to the left of $\chi_{k}^{2}$. Let $t_{1}, t_{1}>t_{0}$, be any time after which $\chi_{i}^{1}$ and $\chi_{i}^{2}$ do not intersect $\chi_{j}^{1}$ and $\chi_{j}^{2}$ for $i \neq j$. We denote by $D_{k}(t)$ the distance between $\chi_{k}^{1}$ and $\chi_{k}^{2}$ at time $t, X_{k}^{ \pm}(t)$ the amount of $k$-rarefaction and $k$-shock waves respectively between $\chi_{k}^{1}$ and $\chi_{k}^{2}$ at time $t$. Then

$$
X_{k}^{+}(t) \leqq \frac{D_{k}(t)}{t-t_{1}}+0(1)\left[Q_{k}\left(t_{0}, t\right)+h_{k}\left(t_{0}, t\right)\right]
$$

where $Q_{k}\left(t_{0}, t\right)$ is the amount of interaction between $t_{0}$ and $t$ and $h_{k}\left(t_{0}, t\right)$ is the amount of $i$-th waves crossing $\chi_{k}^{1}\left(\chi_{k}^{2}\right)$ for all $i>k(i<k)$ between $t_{0}$ and $t$.

\section{§5. Initial Value Problems}

The main purpose of this section is to investigate the asymptotic behavior of the solution $U(x, t)$ of the initial value problem (1.1), (1.2). We assume that the 
characteristic speeds $\lambda_{i}(U(x, t))$ are strictly separated, i.e. there exist $\mu_{i}$, $i=0,1,2, \ldots, n$, and a positive constant $\delta$ such that

$$
\begin{aligned}
& \mu_{0}<\min _{(x, t)} \lambda_{1}(U(x, t))-\delta, \\
& \max _{(x, t)} \lambda_{i}(U(x, t))+\delta<\mu_{i}<\min _{(x, t)} \lambda_{i+1}(U(x, t))-\delta, \\
& i=1,2, \ldots, n-1, \\
& \max _{(x, t)} \lambda_{n}(U(x, t))+\delta<\mu_{n} .
\end{aligned}
$$

We will investigate the asymptotic shape of $U(x, t)$ in each $i$-th primary region $\Omega_{i}$ defined as

$$
\begin{aligned}
\Omega_{0} & =\left\{(x, t) \mid \frac{x}{t}<\mu_{0}\right\}, \\
\Omega_{i} & =\left\{(x, t) \mid \mu_{i-1}<\frac{x}{t}<\mu_{i}\right\}, \quad i=1,2, \ldots, n, \\
\Omega_{n+1} & =\left\{(x, t) \mid \mu_{n}<\frac{x}{t}\right\} .
\end{aligned}
$$

We set

$W_{i, j}^{+}(t)=$ total amount of $i$-rarefaction waves contained in $\Omega_{j}$ at time $t$,

$W_{i, j}^{-}(t)=$ total amount of $i$-shock waves contained in $\Omega_{j}$ at time $t, i=1,2, \ldots, n$, $j=0,1, \ldots, n+1$.

Since the total amount of interactions is finite and $U(\cdot, t)$ has uniformly bounded total variation for each $t$, for any given $\varepsilon>0$, there exist $t_{0}=t_{0}(\varepsilon)>0$ and $M=M(\varepsilon)>0$ such that for any $t \geqq t_{0}$,

$$
\begin{aligned}
& Q\left(t_{0}, t\right)=Q\left\{(x, \tau) \mid t_{0} \leqq \tau \leqq t\right\}<\varepsilon, \\
& \text { total } \operatorname{var}_{x}\left\{U\left(x, t_{0}\right)|| x \mid \geqq M\right\}<\varepsilon .
\end{aligned}
$$

We denote by $\chi_{k}^{1}$ and $\chi_{k}^{2}, k=1,2, \ldots, n$, the $k$-th generalized characteristics issued from $\left(-M, t_{0}\right)$ and $\left(M, t_{0}\right)$ respectively. The quantities $D_{k}(t), X_{k}^{ \pm}(t)$ and $t$, are defined as in Section 4 for each given $\chi_{k}^{1}$ and $\chi_{k}^{2}$ and $t_{0}$. In what follows, $0(1)$ are bounded functions independent of $t$ and $\varepsilon$.

Lemma 5.1. Let $\Gamma_{i}, i=1,2, \ldots, n$, be the region between $\chi_{i}^{1}$ and $\chi_{i}^{2}$ and $\Lambda_{0}$ the region left of $\chi_{1}^{1}, \Lambda_{i}, i=1,2, \ldots, n-1$, the region between $\chi_{i}^{2}$ and $\chi_{i+1}^{1}$, and $\Lambda_{n}$ the region right of $\chi_{n}^{2}$. Then for any $t \geqq t_{1}, j=1,2, \ldots, n, i=0,1,2, \ldots, n$.

(i) The amount of $j$-waves outside $\Gamma_{j}$ at time $t$ is $0(1) \varepsilon$.

(ii) The total variation of $U$ in regions $\Lambda_{i}$ at time $t$ is $0(1) \varepsilon$.

(iii) For any $\left(x_{1}, t_{1}\right)$ and $\left(x_{2}, t_{2}\right)$ in $\Lambda_{i},\left|U\left(x_{1}, t_{1}\right)-U\left(x_{2}, t_{2}\right)\right|=0(1) \varepsilon$.

(iv) $X_{j}^{+}(t) \leqq \frac{D_{j}(t)}{t-t_{1}}+0(1) \varepsilon$. 
Proof. We apply the conservation law (3.1) to the region right of $\chi_{k}^{2}$ to obtain that the amount of $i$-waves, $i<k$, which cross $\chi_{k}^{2}$ is less than the total variation of $U\left(x, t_{0}\right)$ for $x>M$, plus the amount of interactions in the region. Similar estimates hold for the amount of $i$-waves, $i>k$, which cross $\chi_{k}^{1}$. Thus (i) follows from (5.2); and as a direct consequence of (i), we have (ii) and (iii). Finally, (iv) follows from (i) and estimate (4.7) Q.E.D.

Theorem 5.2. The amount of $i$-waves, $i=1,2, \ldots, n$, in the region $\Omega_{j}, j=0,1,2, \ldots, n+1$, $i \neq j$, at time $t$ approaches zero as $t \rightarrow+\infty$.

Proof. According to Lemma 5.1, since $\varepsilon$ is arbitrary, we need only to show that $\Gamma_{j}$ is contained in $\Omega_{j}$ for large $t$, but this is obvious from the definitions of $\Omega_{j}$ and $\Gamma_{j}$. Q.E.D.

Lemma 5.3. Suppose that $i=\beta_{1}, \ldots, \beta_{n-p}$, i.e. $r_{i} \cdot \nabla \lambda_{i} \equiv 0$. Then for any $\left(x_{k}, t_{k}\right)$ in $\Lambda_{k}$, $k=i, i-1$,

(i) $\lambda_{o}\left(U\left(x_{i}, t_{i}\right)\right)=\lambda_{i}\left(U\left(x_{i-1}, t_{i-1}\right)\right)+0(1) \varepsilon$,

(ii) $U\left(x_{i}, t_{i}\right) \in T_{i}\left(U\left(x_{i-1}, t_{i-1}\right)\right)+0(\varepsilon)$.

Proof. Since $\lambda_{i}$ changes value only across $j$-waves, $j \neq i$, (i) is a consequence of Lemma 5.1 , (i). We note that $T_{i}$ are integral waves of the vector field $r_{i} \cdot \nabla$, thus (ii) follows also from Lemma 5.1, (i). Q.E.D.

Lemma 5.4. Suppose that $i=\alpha_{1}, \ldots, \alpha_{p}$, i.e. $r_{i} \cdot \nabla \lambda_{i} \neq 0$, and $\lambda_{i}\left(U\left(x_{i}, t_{i}\right)\right)$ $\leqq \lambda_{i}\left(U\left(x_{i-1}, t_{i-1}\right)\right)-k \varepsilon$ for some $\left(x_{i}, t_{i}\right) \in \Lambda_{i}$ and $\left(x_{i-1}, t_{i-1}\right) \in \Lambda_{i-1}$ and $k>0$. Then there exists a constant $k_{0}$ independent of $t$ and $\varepsilon$ such that for $t$ sufficiently large and $k>k_{0}$,

(i) $X_{i}^{+}(t)=0(1) \varepsilon$,

(ii) $\chi_{i}^{1}$ and $\chi_{i}^{2}$ coalesce to form an $i$-shock with strength $\lambda_{i}\left(U\left(x_{i}, t_{i}\right)\right)-\lambda_{i}\left(U\left(x_{i-1}\right.\right.$, $\left.\left.t_{i-1}\right)\right)+0(1) \varepsilon$.

Proof. We will use the notation in Section 4 [cf. (4.1), (4.2)]. Since $\tilde{X}_{i}(t)=0(1) \varepsilon$ from Lemma 5.1, (i) and $\lambda_{i}^{+2}(t)=\lambda_{i}\left(U\left(x_{i}, t_{i}\right)\right)+0(1) \varepsilon, \lambda_{i}^{-1}(t)=\lambda_{i}\left(U\left(x_{i-1}, t_{i-1}\right)+0(1) \varepsilon\right.$ from Lemma 5.1, (iii), we have from (4.2) that for some $\theta \in(0,1)$,

$$
\begin{aligned}
\dot{D}_{i}(t) & \leqq \theta\left[X_{i}^{+}(t)+X_{i}^{-}(t)\right]+(1-\theta)\left[\lambda _ { i } \left(U\left(x_{i}, t_{i}\right)\right.\right. \\
& \left.-\lambda_{i}\left(U\left(x_{i-1}, t_{i-1}\right)\right)\right]+0(1) \varepsilon, \quad t \geqq t_{1} .
\end{aligned}
$$

Thus it follows from Lemma 5.1, (iv) that

$$
\begin{aligned}
\dot{D}_{i}(t) & \leqq \theta \frac{D_{i}(t)}{t-t_{1}}+(1-\theta)\left[\lambda _ { i } \left(U\left(x_{i}, t_{i}\right)\right.\right. \\
& \left.-\lambda_{i}\left(U\left(x_{i-1}, t_{i-1}\right)\right)\right]+0(1) \varepsilon, \quad t \geqq t_{1} .
\end{aligned}
$$

If we set

$$
H_{i}(t)=D_{i}(t)-\left[\lambda_{i}\left(U\left(x_{i}, t_{i}\right)\right)-\lambda_{i}\left(U\left(x_{i-1}, t_{i-1}\right)\right)\right]\left(t-t_{1}\right),
$$

then (5.3) yields

$$
\dot{H}_{i}(t) \leqq \theta \frac{H_{i}(t)}{t-t_{1}}+0(1) \varepsilon, \quad t \geqq t_{1} .
$$


This is a differential inequality which can be easily solved to yield

$$
\begin{aligned}
D_{i}(t) \leqq & \operatorname{const}\left(t-t_{i}\right)^{\theta}+\left[\lambda_{i}\left(U\left(x_{i}, t_{i}\right)\right)-\lambda_{i}\left(U\left(x_{i-1}, t_{i-1}\right)\right)\right] \\
& \cdot\left(t-t_{1}\right)+0(1) \varepsilon\left(t-t_{1}\right), \quad t \geqq t_{1} .
\end{aligned}
$$

Since $\theta \in(0,1)$, it follows from (5.4) that if $k_{0}$ is so chosen that $k_{0}>0(1)$ on RHS of (5.4), then $D_{i}(t)=0$ for $t$ large. Thus the lemma follows from Lemma 5.1. Q.E.D.

Lemma 5.5. Suppose that $i=\alpha_{1}, \ldots, \alpha_{p}$, i.e. $r_{i} \cdot \nabla \lambda_{i} \neq 0$, and $\lambda_{i}\left(U\left(x_{i}, t_{i}\right)\right)>\lambda_{i}\left(U\left(x_{i-1}\right.\right.$, $\left.\left.t_{i-1}\right)\right)-0(1) \varepsilon$ for some $0(1)>0$. Then for $t$ sufficiently large,

(i) $\left|X_{i}^{-}(t)\right|=0(1) \varepsilon$,

(ii) $U\left(x_{i}, t_{i}\right) \in R^{+}\left(U\left(x_{i-1}, t_{i-1}\right)\right)+0(1) \varepsilon$.

Proof. It follows from Lemma 5.1, (iv) and estimate (5.4) that

$$
\begin{aligned}
X_{i}^{+}(t) & \leqq \operatorname{const}\left(t-t_{1}\right)^{\theta-1}+\lambda_{i}\left(U\left(x_{i}, t_{i}\right)\right) \\
& -\lambda_{i}\left(U\left(x_{i-1}, t_{i-1}\right)\right)+0(1) \varepsilon, \quad t \geqq t_{1},
\end{aligned}
$$

and so for $t$ large,

$$
X_{i}^{+}(t) \leqq \lambda_{i}\left(U\left(x_{i}, t_{i}\right)\right)-\lambda_{i}\left(U\left(x_{i-1}, t_{i-1}\right)\right)+0(1) \varepsilon .
$$

Since

$$
X_{i}^{+}(t)+X_{i}^{-}(t)+0(1) \varepsilon=\lambda_{i}\left(U\left(x_{i}, t_{i}\right)\right)-\lambda_{i}\left(U\left(x_{i-1}, t_{i-1}\right)\right)
$$

as is easily seen from Lemma 5.1, it follows from (5.5) that

$$
\begin{aligned}
& X_{i}^{+}(t)=\lambda_{i}\left(U\left(x_{i}, t_{i}\right)\right)-\lambda_{i}\left(U\left(x_{i-1}, t_{i-1}\right)\right)+0(1) \varepsilon, \\
& X_{i}^{-}(t)=0(1) \varepsilon .
\end{aligned}
$$

The lemma follows from Lemma 5.1. and (5.6). Q.E.D.

Lemma 5.6. Suppose that the Riemann problem(1.1),(1.3) is solved by centered $i$-waves $\left(U_{i-1}, U_{i}\right), i=1, \ldots, n$. Then for any $\left(x_{i}, t_{i}\right) \in \Lambda_{i}$, we have

$$
\left|U\left(x_{i}, t_{i}\right)-U_{i}\right|=0(1) \varepsilon
$$

Proof. It follows from Lemma 5.3, (ii), Lemma 5.4, (ii) and Lemma 5.5, (ii) that there exist $\tilde{U}_{i}, i=0,1, \ldots, n$, such that

$$
\begin{aligned}
& \left|\tilde{U}_{i}-U\left(x_{i}, t_{i}\right)\right|=0(1) \varepsilon, \\
& \tilde{U}_{i} \in T\left(\tilde{U}_{i-1}\right) .
\end{aligned}
$$

Thus $\left(\tilde{U}_{i-1}, \tilde{U}_{i}\right), i=1,2, \ldots, n$, solves the Riemann problem with data $\left(\tilde{U}_{0}, \tilde{U}_{n}\right)$. But the above inequality and Lemma 5.1 , (iii) imply that $\left|\tilde{U}_{0}-U(-\infty)\right|+\left|\tilde{U}_{n}-U(+\infty)\right|$ $=0(1) \varepsilon$. Because the solution of the Riemann problem depends differentiably on its data, we have proved the lemma. Q.E.D.

Theorem 5.7. Suppose that the Riemann problem (1.1), (1.3) is solved by $i$-th centered waves $\left(U_{i-1}, U_{i}\right), i=1,2, \ldots, n$. Then

(i) $U(x, t) \rightarrow U_{i}$ as $t \rightarrow+\infty$ for $\frac{x}{t}=\mu_{i}$. 
(ii) If $r_{i} \cdot \nabla \lambda_{i} \neq 0$, i.e. $i=\alpha_{1}, \alpha_{2}, \ldots, \alpha_{p}$, and $\left(U_{i-1}, U_{i}\right)$ is a centered rarefaction wave, i.e. $\lambda_{i}\left(U_{i-1}\right) \leqq \lambda_{i}\left(U_{i}\right)$, then the amount of $i$-shock waves in $\Omega_{i}$ approaches zero as $t \rightarrow$ $+\infty$ and $U(x, t)$ approaches the centered rarefaction wave $\left(U_{i-1}, U_{i}\right)$ pointwise in $\Omega_{i}$ as $t \rightarrow+\infty$.

(iii) If $r_{i} \cdot \nabla \lambda_{i} \neq 0$, i.e. $i=\alpha_{1}, \alpha_{2}, \ldots, \alpha_{p}$ and $\left(U_{i-1}, U_{i}\right)$ is centered shock wave, i.e. $\lambda_{i}\left(U_{i-1}\right)>\lambda_{i}\left(U_{i}\right)$, then there exists an $i$-shock wave in $\Omega_{i}$ which approchaes $\left(U_{i-1}, U_{i}\right)$ both in strength and speed and, moreover, the total variation of the solution in $\Omega_{i}$ outside of this shock wave approaches zero as $t \rightarrow+\infty$.

(iv) If $r_{i} \cdot \nabla \lambda_{i} \equiv 0$, i.e. $i=\beta_{1}, \beta_{2}, \ldots, \beta_{n-p}$, then in $\Omega_{i}, \lambda_{i}(U(x, t)) \rightarrow \lambda_{i}\left(U_{i-1}\right)=\lambda_{i}\left(U_{i}\right)$ as $t \rightarrow+\infty$ and the distance between $U(x, t),(x, t) \in \Omega_{i}$, and $T\left(U_{i-1}\right)=T\left(U_{i}\right)$ approaches zero uniformly as $t \rightarrow+\infty$.

Proof. It is easy to see that given any $\varepsilon>0$ and associated $\Gamma_{i}, i=0,1,2, \ldots, n$, the point $(x, t)$ with $\frac{x}{t}=\mu_{i}$ belongs to $\Gamma_{i}$ if $t$ is sufficiently large. Thus (i) of the theorem follows from Lemma 5.6 and the arbitrariness of $\varepsilon$. Similarly, the first half of (ii) follows from Lemma 5.5, (i) and Lemma 5.6. We now prove that $U(x, t)$ approaches the centered rarefaction wave $\left(U_{i-1}, U_{i}\right)$ in $\Omega_{i}$.

Given any $\varepsilon>0$, we construct $\chi_{i}^{1}$ and $\chi_{i}^{2}$ as above. By Lemma 5.5, (i) there exists $t_{2} \geqq t_{1}$ such that $\left|X_{i}^{-}(t)\right| \leqq 0(1) \varepsilon$ for $t \geqq t_{2}$. Thus it follows from Lemma 5.6 that the speeds of $\chi_{k}^{1}$ and $\chi_{k}^{2}$ for $t \geqq t_{2}$ are $\lambda_{i}\left(U_{i-1}\right)+0(1) \varepsilon$ and $\lambda_{i}\left(U_{i}\right)+0(1) \varepsilon$, respectively. Thus for $t \geqq t_{2}+D_{i}\left(t_{2}\right)$,

$$
\text { distance }\left\{\chi_{i}^{1}, l_{i}^{1}\right\}+\text { distance }\left\{\chi_{i}^{2}, l_{i}^{2}\right\}=0(1) \varepsilon\left(t-t_{2}\right) \text {, }
$$

where $l_{i}^{j}=\{(x, t)\} \mid \frac{x}{t}=\lambda_{i}\left(U_{i-2+j}\right), j=1,2$, are the edges of the centered wave $\left(U_{i-1}, U_{i}\right)$. For $(x, t) \in \Lambda_{i-1}$, it follows from (5.7), Lemma 5.6, and the structure of centered rarefaction waves that

$$
\begin{aligned}
\left|U^{*}(x, t)-U(x, t)\right| & =\left|U^{*}(x, t)-U_{i-1}\right|+\left|U_{i-1}-U(x, t)\right| \\
& =0(1) \varepsilon \frac{t-t_{2}}{t}+0(1) \varepsilon=0(1) \varepsilon
\end{aligned}
$$

where $U^{*}(x, t)$ denotes the centered wave $\left(U_{i-1}, U_{i}\right)$. Similarly, for $(x, t) \in \Lambda_{i}$,

$$
\left|U^{*}(x, t)-U(x, t)\right|=0(1) \varepsilon .
$$

For any $(x, t) \in \Gamma_{i}, t \geqq t_{2}+D_{i}\left(t_{2}\right)$, we can choose $\left(x^{*}, t\right)$ between $l_{i}^{1}$ and $l_{i}^{2}$ such that $\left|U^{*}\left(x^{*}, t\right)-U(x, t)\right|=0(1) \varepsilon$ as is easily seen from Lemmas 5.1 and 5.6. Through $(x, t)$ we draw an $i$-th generalized characteristic $\chi$ backward in time. If $\chi$ meets $\chi_{i}^{1}$ and $\chi_{i}^{2}$ we continue $\chi$ with $\chi_{i}^{1}$ or $\chi_{i}^{2}$. Since $\chi$ may change speed only due to shock waves entering $\chi$ or $j$-waves, $i \neq j$, crossing $\chi$, one sees that for $t \geqq t_{2}, \chi$ has speed $\lambda_{i}(U(x, t))$ $+0(1) \varepsilon$. As a result we see that

$$
\left|x^{*}-x\right|=0(1) \varepsilon\left(t-t_{2}\right) \text {. }
$$

Similarly we may draw an $i$-th generalized characteristic $\chi^{*}$ through $\left(x^{*}, t\right)$ and apply estimate (4.7) for $\chi$ and $\chi^{*}$ [cf. Lemma 5.1, (iv)] together with (5.10) to yield that the total amount of $i$-rarefaction waves between $\chi$ and $\chi^{*}$ is $0(1) \varepsilon$. This along 
with Lemma 5.5, (i), yields that for $(x, t) \in \Gamma_{i}, t$ large

tot $\operatorname{var}\left\{U(\cdot, t)\right.$ between $x$ and $\left.x^{*}\right\}=0(1) \varepsilon$

and so

$\left|U^{*}(x, t)-U(x, t)\right|=0(1) \varepsilon$.

Thus it follows from (5.8), (5.9), (5.11) and the arbitrariness of $\varepsilon$ that $U(x, t) \rightarrow U^{*}(x, t)$ is uniformly in $\Omega_{i}$ as $t \rightarrow+\infty$.

Statements (iii) and (iv) of the theorem follow from Lemmas 5.3 and 5.4 by analogous arguments. We omit the details. Q.E.D.

The following corollary is a direct consequence of the above theorem. We omit the proof.

Corollary. Suppose that $U(-\infty)=U(+\infty)$ and let $\psi$ be any i-Riemann invariant, $i=\beta_{1}, \beta_{2}, \ldots, \beta_{n-p}$, i.e. $\psi$ is constant along all $T_{i}$ curves for all $i \in\left\{\beta_{1}, \beta_{2}, \ldots, \beta_{n-p}\right\}$

$$
\text { tot } \operatorname{var}\{\psi(x, t) \mid-\infty<x<\infty\} \rightarrow 0 \text { as } t \rightarrow+\infty \text {. }
$$

If $p=n$, i.e., all characteristic fields are genuinely nonlinear, then

$$
\text { tot } \operatorname{var}\{U(x, t) \mid-\infty<x<\infty\} \rightarrow 0 \text { as } t \rightarrow+\infty .
$$

\section{§6. Initial-Boundary Value Problems}

In this section we investigate the large-time behavior of solutions of (1.1) defined in the quadrant $x \geqq 0, t \geqq 0$. We will illustrate our basic ideas for gas equations:

$$
\begin{aligned}
& \left.\begin{array}{l}
u_{t}+p_{x}=0, \\
v_{t}-u_{x}=0, \\
E_{t}+(p u)_{x}=0, \quad p=p(s, e)=p(s, v), \quad E=e+\frac{1}{2} u^{2}, \\
p_{v}(s, v)<0, \quad p_{v v}(s, v)>0,
\end{array}\right\} \\
& \begin{array}{l}
(u, v, E)(x, 0)=\left(u_{0}, v_{0}, E_{0}\right)(x), \quad x \geqq 0, \\
u(0, t)=u_{b}(t), \quad t \geqq 0,
\end{array}
\end{aligned}
$$

or,

$$
p(0, t)=p_{b}(t), \quad t \geqq 0 .
$$

It follows from the estimates in Liu [15] that there exists a finite amount of interactions $Q_{0}$ in the interior $t \geqq 0, x>0$ and $Q_{1}$ on the boundary $x=0$ provided that either the data (6.2), (6.3) have small total variation or the gas is polytropic, i.e. $p(s, v)=$ const $\exp \left(\frac{(\gamma-1) s}{R}\right) v^{-\gamma}, 1<\gamma \leqq 5 / 3$, and $(\gamma-1)$ times the total variation of the data (6.2), (6.3) is small, (see also Section 3). Given any region $\Lambda$ in the quadrant which intersects the boundary $x=0$ from $t=a$ to $t=b$, the following approximate conservation laws hold $\left[\mathrm{cf} .(2.7)_{i}\right]$ when $(6.3)_{1}$ is the boundary data

$$
\begin{aligned}
& L_{1}^{ \pm}(\Lambda)=E_{1}^{ \pm}(\Lambda)-R_{1}^{ \pm}(a, b) \mp C_{1}(\Lambda)+0(1)\left[Q_{0}(\Lambda)+Q_{1}(a, b)\right], \\
& L_{3}^{ \pm}(\Lambda)=E_{3}^{ \pm}(\Lambda)+R_{1}^{ \pm}(a, b) \mp C_{3}(\Lambda)+0(1)\left[Q_{0}(\Lambda)+Q_{1}(a, b)+B_{3}^{ \pm}(a, b)\right]
\end{aligned}
$$


and when $(6.3)_{2}$ is the boundary data then the first equation in (6.4) is replaced by

$$
L_{3}^{ \pm}(\Lambda)=E_{3}^{ \pm}(\Lambda)-R_{1}^{ \pm}(a, b) \mp C_{3}(\Lambda)+0(1)\left[Q_{0}(\Lambda)+Q_{1}(a, b)+B_{3}^{ \pm}(a, b)\right] .
$$

Here $R_{1}^{ \pm}(a, b)$ denotes the amount of 1 -waves hitting the line $x=0$ between time $a$ and $b$, and $B_{3}^{ \pm}(a, b)$ the amount of 3-waves issuing from $x=0$ due to the boundary data (6.3).

Given any $\varepsilon>0$, we choose $t_{0}, M$ so large that $Q_{2}(\Lambda)+Q_{1}\left(t_{0}, \infty\right) \leqq \varepsilon$ for $\Lambda=\left\{(x, t) \mid t \geqq t_{0}\right\}$, and $\left|B_{3}^{ \pm}\left(t_{0}, \infty\right)\right|<\varepsilon$, tot $\operatorname{var}\{U(x, t), x \geqq M\}<\varepsilon$. Through $\left(M, t_{0}\right)$ we construct a 1-characteristic $\chi$ which intersects $x=0$ at time $t_{1}$. Applying (6.4), to the region right of $\chi$, we find that for any $t \geqq t_{1}$

$$
\left|X_{1}^{ \pm}(t)\right|=0(1) \varepsilon, \quad\left|R_{1}^{ \pm}(t, \infty)\right|=0(1) \varepsilon,
$$

and so, $(6.4)_{3}$ becomes

$$
L_{3}^{ \pm}(\Lambda)=E_{3}^{ \pm}(\Lambda) \mp C_{3}(\Lambda)+0(1) \varepsilon
$$

for all $\Lambda$ in the region $\left\{(x, t) \mid t \geqq t_{1}\right\}$. That is, for $t$ sufficiently large, the amount of 1 waves is small and thus we may use the techniques used in the last section to prove the following theorem whose proof is omitted:

Theorem 6.1. Suppose that either TV is sufficiently small or the gas is polytropic with exponent $\gamma, 1<\gamma<5 / 3$ and $(\gamma-1) T V$ is sufficiently small. Here TV is the total variation of the data (6.2),(6.3). Then the initial-boundary value problems have a global solution $(u, v, E)(x, t)$ which approaches the solution $\left(u^{*}, v^{*}, E^{*}\right)(x, t)$ of $(6.1)$ such that

$$
\begin{aligned}
& (u, v, E)(x, 0)=\left(u_{0}, v_{0}, E_{0}\right)(+\infty), \quad x \geqq 0, \\
& u(0, t)=u_{b}(+\infty), \quad t \geqq 0,
\end{aligned}
$$

or,

$$
p(0, t)=p_{b}(+\infty), \quad t \geqq 0 .
$$

More precisely, if $\left(u^{*}, v^{*}, E^{*}\right)(x, t)$ is a rarefaction wave or a constant, then shock waves decay and $(p, u)(x, t)$ approaches $\left(p^{*}, u^{*}\right)(x, t)$ pointwise as $t \rightarrow+\infty$, and if $\left(u^{*}, v^{*}, E^{*}\right)(x, t)$ is a shock wave, then a shock wave emerges from $(u, v, E)(x, t)$ for $t$ large such that the shock wave approaches the shock wave $\left(u^{*}, v^{*}, E^{*}\right)(x, t)$ both in speed and strength and outside the shock wave $(u, p)(\cdot, t)$ has total variation approaching zero as $t \rightarrow+\infty$.

\section{References}

1. Bakhrarov, N.: On the existence of regular solutions in the large for quasilinear hyperbolic systems. Zh. Vychisi. Mat. Mathemat. Fiz. 10, 969-980 (1970)

2. Ballou,D.P.: The structure and asymptotic behavior of compression waves. Arch. Rat. Mech. Anal. 56, 170-180 (1974)

3. DiPerna, R.J.: Global solutions to a class of nonlinear hyperbolic systems of equations. Comm. Pure Appl. Math. 26, 1-28 (1973)

4. DiPerna, R.J.: Decay of solutions of hyperbolic systems of conservation laws with a convex extension, preprint

5. Glimm,J.: Solutions in the large for nonlinear hyperbolic systems of equations. Comm. Pure Appl. Math. 18, 697-715 (1965) 
6. Glimm,J., Lax,P.D.: Decay of solutions of systems of nonlinear hyperbolic conservation laws. Amer. Math. Soc. 101 (1970)

7. Greenberg,J.M.: On the interaction of shock and simple waves of the same family II. Arch. Rat. Mech. Anal. 51, 209-217 (1973)

8. Greenberg,J.M.: Asymptotic behavior of solutions to the quasilinear wave equation, Lecture notes in mathematics, Vol. 446. Partial differential equations and related topics. Berlin-HeidelbergNew York: Springer 1975

9. Kuznetsov, N.N., Tupshiev,V.A.: A certain generalization of a theorem of Glimm. Dokl. Akad. Nauk SSSR 221, 287-290 (1975)

10. Lax,P.D.: Hyperbolic systems of conservation laws II. Comm. Pure Appl. Math. 10, 537-566(1957)

11. Lax,P.D.: Shock waves and entropy. Contribution to nonlinear functional analysis (ed. E. A.Zarantonello), pp. 603-634. New York: Academic Press 1971

12. Liu, T.P.: Existence and uniqueness theorems for Riemann problems. Trans. Amer. Math. Soc. 212, $375-382(1975)$

13. Liu,T.P.: Shock waves in the nonisentropic gas flow. J. Diff. Eq. 22, $442-452$ (1976)

14. Liu,T.P.: Solutions in the large for the equations of nonisentropic gas dynamics. Indiana Univ. Math. J. 26, 147-178 (1977)

15. Liu,T.P.: Initial-boundary value problems for gas dynamics. Arch. Rat. Mech. Anal. (to appear)

16. Liu, T.P.: Asymptotic behavior of solutions of general system of nonlinear hyperbolic conservation laws, preprint

17. Nishida,T.: Global solutions for an initial boundary value problem of a quasilinear hyperbolic system. Proc. Japan Acad. 44, 642-646 (1968)

18. Nishida,T., Smoller,J. A. : Solutions in the large for some nonlinear hyperbolic conservation laws. Comm. Pure Appl. Math. 26, 183-200 (1973)

19. Nishida,T., Smoller,J. A. : Mixed problems for nonlinear conservation laws. J. Diff. Eq. 26, 244-269 (1977)

20. Smith, R.: The Riemann problem of gas equations. Ph.D. Thesis, Univ. of Michigan, 1976

21. Smoller,J.A.: On the solution of the Riemann problem with general step data for an extended class of hyperbolic systems. Mich. Math. J. 16, 201-210 (1969)

22. Smoller,J. A. : A uniqueness theorem for Riemann problems. Arch. Rat. Mech. Anal. 33, 110-115 (1969)

23. Smoller,J.A., Johnson,J.L.: Global solutions for an extended class of hyperbolic systems of conservation laws. Arch. Rat. Mech. Anal. 32, 169-189 (1969)

Communicated by J. Glimm

Received March 29, 1977 
